\title{
Aspects of vascularization in Multi-Organ-Chips
}

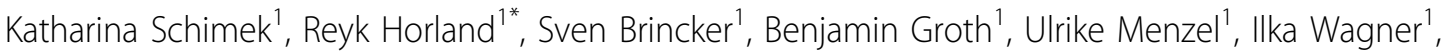 \\ Eva-Maria Materne', Gerd Lindner ${ }^{1}$, Alexandra Lorenz ${ }^{1}$, Silke Hoffmann ${ }^{1}$, Mathias Busek², Frank Sonntag ${ }^{2}$, \\ Udo Klotzbach², Roland Lauster ${ }^{1}$, Uwe Marx ${ }^{1,3}$ \\ From 23rd European Society for Animal Cell Technology (ESACT) Meeting: Better Cells for Better Health \\ Lille, France. 23-26 June 2013
}

\section{Background}

Enormous efforts have been made to develop circulation systems for physiological nutrient supply and waste removal of in vitro cultured tissues. These developments are aiming for in vitro generation of organ equivalents such as liver, lymph nodes and lung or even multi-organ systems for substance testing, research on organ regeneration or transplant manufacturing. Initially technical perfusion systems based on membranes, hollow fibers or networks of micro-channels were used for these purposes. However, none of the currently available systems ensures long-term homeostasis of the respective tissue over months. This is caused by a lack of in vivo-like vasculature which leads to continuous accumulation of protein sediments and cell debris in the systems. Here, we demonstrate a closed and self-contained circulation system emulating the natural blood perfusion environment of vertebrates at tissue level.

\section{Material and methods}

The Multi-Organ-Chip (MOC) device accommodates two microvascular circuits (Figure 1a). Each circuit is operated by a separate peristaltic on-chip micropump, modified from Wu and co-workers [1]. Microfluidic 3D channels were formed in PDMS by replica molding from master molds and were afterwards closed by bonding to a coverslip by air plasma treatment. To retain PDMS hydrophilicity, channels were filled with culture medium immediately after sealing. To emulate the natural blood perfusion environment, human dermal microvascular endothelial cells (HDMEC) were used. The cells were seeded into the PDMS channels and adhered to all channel walls after subsequent static cultivation on each channel side. Afterwards

\footnotetext{
* Correspondence: reyk.horland@tu-berlin.de

${ }^{1} \mathrm{TU}$ Berlin, Institute of Biotechnology, Faculty of Process Science and Engineering, 13355 Berlin, Germany

Full list of author information is available at the end of the article
}

cells were cultured up to 14 days in PDMS channels under pulsatile flow conditions.

\section{Results}

A miniaturized circulation system has been established over a period of 14 days by fully covering all channels and surfaces of the MOC with human microvascular endothelial cells. By injecting $2 \times 10^{7}$ cells ml $^{-1}$ into the channels, a homogeneous distribution of cells throughout all channels was achieved (Figure 1b). During the following static incubation, cells adhered well to the air plasma treated channel walls. A peristaltic micro-pump was used to create culture medium circulation. After adaption to shear stress, HDMEC showed an elongation and alignment parallel to the flow direction. Three-dimensional reconstitutions of image stacks indicate that cells formed confluent monolayers on all walls of the channels (Figure 1c). During the whole cultivation time they maintained adherence to the channel walls and were positive for Calcein AM viability staining (Figure 1b). After 14 days of culture HDMEC forming the microvascular circuit were positive for ac-LDL uptake (Figure 1d) and expressed the endothelialspecific marker CD31 and von Willebrand Factor (vWF) (Figure 1e).

\section{Conclusion}

A robust procedure applying pulsatile shear stress has been established to cover all fluid contact surfaces of the system with a functional, tightly closed layer of HDMEC.

Long-term cultivation of elongated and flow-aligned HDMEC inside the chip-based microcirculation was demonstrated over a period of 14 days. For such endothelialized microfluidic devices to be useful for substance testing, it is essential to show long-term viability and function in the presence of physiological flow rates as shown here. These artificial vessels are an important approach for systemic substance testing in Multi-Organ-Chips. The miniaturized 


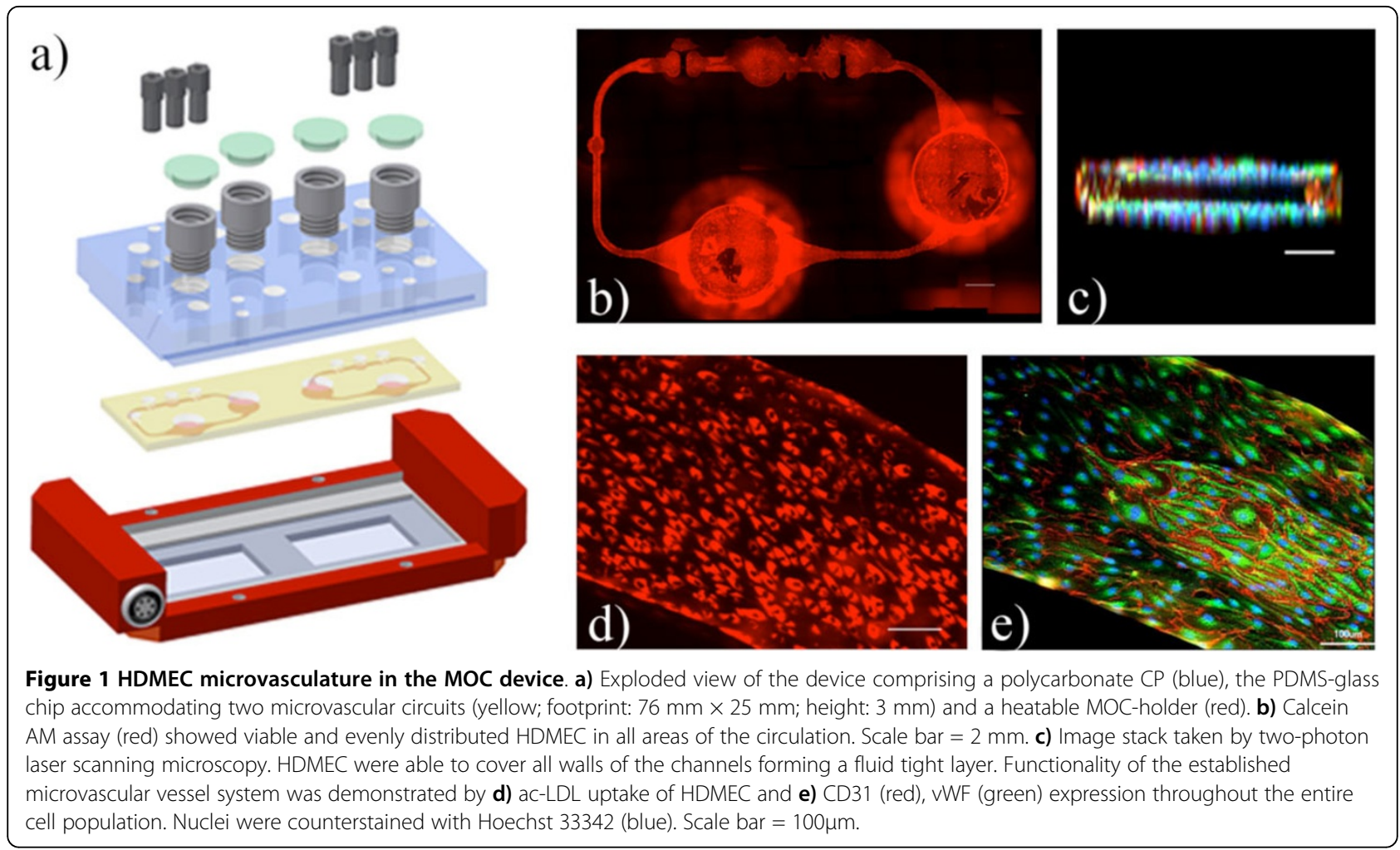

circulation system creates the conditions for circulation of nutrients through the organoid culture chamber, allows for in vivo-like crosstalk between endothelial cells and tissues and prevents clumping inside the channels. Compared with conventional cell culture techniques, a microfluidic-based cell culture may mimic more accurate in vivo-like extracellular conditions, as the culture of cells and organ models in perfused microfluidic systems can improve their oxygen and nutrient supply. This makes it suitable for long-term cultivation and more efficient drug studies. In future, such endothelialized bioreactors might be used for testing vasoactive substances. Finally, the described system can now be used for the establishment of organ-specific capillary networks. Here, we will adhere to our recently published roadmap toward vascularized "human-on-a-chip" models to generate systemic data fully replacing the animals or human beings currently used [2].

\section{Acknowledgements}

The work has been funded by the German Federal Ministry for Education and Research, GO-Bio Grant No. 0315569

\section{Authors' details}

${ }^{1} \mathrm{TU}$ Berlin, Institute of Biotechnology, Faculty of Process Science and Engineering, 13355 Berlin, Germany. ${ }^{2}$ Fraunhofer IWS Dresden, 01277 Dresden, Germany. ${ }^{3}$ Tiss Use GmbH, 15528 Spreenhagen, Germany.

Published: 4 December 2013

\section{References}

1. Wu M-H, Huang S-B, Cui Z, Cui Z, Lee G-B: A high throughput perfusionbased microbioreactor platform integrated with pneumatic micropumps for three-dimensional cell culture. Biomedical microdevices 2008 10:309-319.

2. Marx U, Walles $H$, Hoffmann $S$, Lindner $G$, Horland R, Sonntag $F$, Klotzbach U, Sakharov D, Tonevitsky A, Lauster R: "Human-on-a-chip" developments: a translational cutting-edge alternative to systemic safety assessment and efficiency evaluation of substances in laboratory animals and man? Alternatives to laboratory animals: ATLA 2012, 40:235-257.

doi:10.1186/1753-6561-7-S6-O6

Cite this article as: Schimek et al:: Aspects of vascularization in Multi-Organ-Chips. BMC Proceedings 2013 7(Suppl 6):06.

\section{Submit your next manuscript to BioMed Central and take full advantage of:}

- Convenient online submission

- Thorough peer review

- No space constraints or color figure charges

- Immediate publication on acceptance

- Inclusion in PubMed, CAS, Scopus and Google Scholar

- Research which is freely available for redistribution 